\title{
Importancia de las competencias digitales en los alumnos de nivel medio superior
}

\section{Importance of digital skills in upper secondary level students}

MELO-MORIN, Julia Patriciał** \& AHUMADA-CERVANTES, María de los Ángeles

Instituto Tecnológico Superior de Pánuco, División de Ingeniería en Sistemas Computacionales

ID $1^{\text {st }}$ Author: Julia Patricia, Melo-Morin / ORC ID: 0000-0001-7145-2344, Researcher ID Thomson: I-3412-2018, CVU CONACYT ID: 248185

ID $1^{\text {st }}$ Coauthor: María De Los Ángeles, Ahumada-Cervantes / ORC ID: 0000-0001-8164-2889, CVU CONACYT ID: 825136

DOI: $10.35429 /$ JET.2020.4.12.28.33

Recibido: 25 de Septiembre, 2020; Aceptado 19 de Diciembre, 2020

\section{Resumen}

Las competencias digitales son de gran importancia en el desarrollo de los individuos para afrontar los desafíos tecnológicos que surgen cada día. Con el confinamiento de los estudiantes en sus hogares por el surgimiento del COVID-19, se pone en manifiesto la necesidad de aplicar las competencias digitales tanto de alumnos y profesores, para continuar con el proceso educativo. Existen cinco áreas fundamentales de las competencias digitales de acuerdo al Marco Europeo de Competencias Digitales, desarrollado por el Joint Research Centre (JRC), que incluyen 21 competencias que los individuos deben desarrollar con respecto al uso de las tecnologías de información y de comunicación (Tics). Se aplicó un cuestionario a estudiantes de nivel medio superior de la Escuela de Bachilleres Diurna de Pánuco, Veracruz, para identificar el nivel de competencias digitales que los alumnos tienen, que permitieran apoyar en el desarrollo de sus clases en línea. Este articulo describe los resultados del instrumento aplicado en cada una de las áreas donde se clasifican las competencias digitales.

Competencias Digitales, Tics, Marco Europeo de Competencias Digitales

\begin{abstract}
Digital skills are of great importance in the development of individuals to face the technological challenges that arise every day. With the confinement of students in their homes due to the emergence of COVID-19, is important the need to apply the digital skills of both students and teachers, to continue the educational process. There are five fundamental areas of digital competences according to the European Framework of Digital Competences, developed by the Joint Research Centre (JRC), which include 21 competencies that individuals must develop with respect to the use of information and communication technologies (Tics). A questionnaire was applied to high school students from the Escuela de Bachilleres Diurna de Pánuco, Veracruz, to identify the level of digital skills that students have, which would allow support in the development of their online classes. This article describes the results of the instrument applied in each of the areas where digital skills are classified.
\end{abstract}

Digital Skills, Tics, European Digital Skills Framework

Citación: MELO-MORIN, Julia Patricia \& AHUMADA-CERVANTES, María de los Ángeles. Importancia de las competencias digitales en los alumnos de nivel medio superior. Revista Teoría Educativa. 2020. 4-12: 28-33

\footnotetext{
* Correspondencia del Autor (Correo electrónico: patricia.melo@itspanuco.edu.mx)

$\dagger$ Investigador contribuyendo como primer autor.
} 


\section{Introducción}

El avance constante en el surgimiento de la tecnología, genera cambios en la forma de actuar, de pensar y en la forma de estudiar o trabajar; modificando completamente el estilo de vida, la Unesco describe que las Tics han generado una revolución cambiando forma de hacer las actividades, de aprender y de vivir en general, impactando en todas las acciones que se realizan (UNESCO, 2011).

En el ámbito de la educación, estos avances han modificado la forma de comunicación entre profesores y alumnos, la forma en que el estudiante se relaciona con la información y los contenidos, cambiando la manera de llevar a cabo el proceso de enseñanzaaprendizaje; García Aretio L. (2019), describe que esta innovación científica y tecnológica es disruptiva, ya que rompe con acciones pasadas y modifica nuestras nuevas formas de actuar.

De acuerdo al INEGI en 2017, hay 71.3 millones de usuarios de Internet en México, con una edad de mínimo 6 años (INEGI, 2017) y cada persona se le ha atribuido pertenecer a una generación tecnológica: $\mathrm{X}, \mathrm{Y}, \mathrm{Z}$, milenials, alpha, etc., y en ocasiones se supone que los niños y jóvenes al nacer en una cierta generación, tienen el conocimiento absoluto de la tecnología que se encuentra en ese momento, por el simple hecho de haber nacido y estar rodeados de la tecnología constantemente; pero es de gran importancia identificar exactamente los usos y aplicación que le dan a dicha tecnología, y sí en verdad cuentan con las competencias adecuadas para poder aplicarlas correctamente.

Instituciones como la OCDE, UNESCO, ONU y la Unión Europea, en diferentes foros, han indicado la necesidad y urgencia de que en todos los niveles educativos desarrollen las competencias digitales en los alumnos. Los jóvenes actualmente acceden a contenidos en línea de forma constante, participan activamente en redes sociales estableciendo un medio de comunicación actual, en ocasiones no saben diferenciar entre lo real y lo virtual, forman parte de diferentes comunidades virtuales, hacen gran uso de materiales multimedia, por lo que no les gusta leer mucho texto, para ellos su identidad digital es algo natural y forma parte de su vida.
Las competencias digitales son "La capacidad de usar el conocimiento y las destrezas relacionadas al desarrollo de elementos y procesos; haciendo uso de éstas (conocimientos, habilidades y aptitudes) que permiten utilizar de manera eficaz y eficiente los instrumentos y recursos tecnológicos" (UNESCO, 2011).

Las competencias digitales permiten la obtención de la información, la comunicación, la creación de nuevos contenidos, aplicar medidas de seguridad a la información y la resolución de problemas utilizando las tecnologías de la información y la comunicación, incluyen acciones que van más allá de la utilización de la computadora.

\section{Antecedentes}

Existen investigaciones que relacionan el uso de la tecnología y las competencias digitales. Lozano Zapata (2017), realizo una tesis donde demuestra que los usos de las Tics se relacionan en el desarrollo de competencias digitales en los alumnos del Instituto de Educación Superior Tecnológico Simón Bolívar, en Lima Perú, donde se elaboraron instrumentos que incluían las diferentes áreas de las competencias y se aplicó a estudiantes de la carrera profesional de Computación e Informática.

Además, un estudio realizado por Facebook México y la empresa Failure Institute, describe la experiencia de 200 empresarios que emprendieron un negocio y fracasaron, donde identificaron las competencias digitales en los empresarios, impactando la falta de ellas en el fracaso de los negocios (2018).

\section{Metodología}

Este articulo describe cual es el conocimiento y aplicación de la tecnología digital en los estudiantes de nivel medio superior, para considerar su nivel de competencia digital.

El estudio se basó en un cuestionario que se aplicó a los alumnos de la escuela de Bachilleres Diurna de Pánuco vía google, incluyendo diferentes preguntas de acuerdo a las 21 competencias digitales, identificadas por el Marco Europeo clasificándolas en 5 áreas, tal como se describen en la Tabla 1. 


\begin{tabular}{|c|c|}
\hline \multicolumn{2}{|c|}{\begin{tabular}{l|l}
1.1 & $\begin{array}{l}\text { Navegación, búsqueda y filtrado de datos } \\
\text { información y contenido digital. }\end{array}$
\end{tabular}} \\
\hline 1.2 & $\begin{array}{l}\text { Evaluación de datos, información y contenidos } \\
\text { digitales }\end{array}$ \\
\hline 1.3 & $\begin{array}{l}\text { Gestión de datos, información y contenidos } \\
\text { digitales }\end{array}$ \\
\hline \multicolumn{2}{|r|}{ Área 2. Comunicación y colaboración. } \\
\hline 2.1 & Interactuar a través de las tecnologías digitales \\
\hline 2.2 & Compartir a través de tecnologías digitales. \\
\hline 2.3 & $\begin{array}{l}\text { Participar en la ciudadanía a través de las } \\
\text { tecnologías digitales. }\end{array}$ \\
\hline 2.4 & Colaboración a través de tecnologías digitales. \\
\hline 2.5 & Netiquette, o etiqueta digital. \\
\hline 2.6 & Gestión de la identidad digital. \\
\hline \multicolumn{2}{|r|}{ Área 3. Creación de contenidos digitales. } \\
\hline 3.1 & Desarrollando contenidos digitales. \\
\hline 3.2 & $\begin{array}{llll}\begin{array}{l}\text { Integración } \\
\text { digitales. }\end{array} & \text { reelaboración de contenidos } \\
\end{array}$ \\
\hline 3.3 & Derechos de autor y licencias. \\
\hline 3.4 & Programación. \\
\hline \multicolumn{2}{|r|}{ Área 4. Seguridad. } \\
\hline 4.1 & Dispositivos de protección. \\
\hline 4.2 & Protección de datos personales y privacidad. \\
\hline 4.3 & Protección de la salud y el bienestar. \\
\hline 4.4 & Proteger el medio ambiente. \\
\hline \multicolumn{2}{|r|}{ Área 5. Resolución de problemas. } \\
\hline 5.1 & Resolución de problemas técnicos. \\
\hline 5.2 & $\begin{array}{l}\begin{array}{l}\text { Identificación de necesidades y respuestas } \\
\text { tecnológicas. }\end{array} \\
\end{array}$ \\
\hline 5.3 & Uso creativo de las tecnologías digitales. \\
\hline 5.4 & $\begin{array}{l}\text { Identificación de brechas en las competencias } \\
\text { digitales. }\end{array}$ \\
\hline
\end{tabular}

Tabla 1 Relación de competencias digitales Fuente: Joint Research Centre (JRC)

\section{Resultados}

Se aplicó el cuestionario a 165 estudiantes, de los cuales el $73.9 \%$ son del sexo femenino y el $26.1 \%$ son del sexo masculino.

Considerando las cinco áreas donde se clasifican las competencias digitales, se describen los resultados de algunas preguntas planteadas donde los alumnos indican las ventajas y desventajas del uso de las Tics en el proceso de enseñanza-aprendizaje; los estudiantes consideran que las ventajas en mayor significancia son: Acceso a la información, Creación de contenidos, Publicación de información, Diversidad de metodología, flexibilidad de tiempos y Equipamiento de espacios, tal como se muestra en la Gráfica 1.

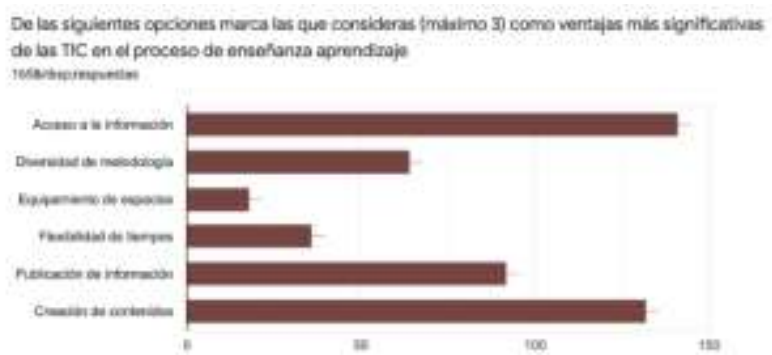

Gráfico 1 Ventajas del uso de las Tics.

Fuente: Elaboración Propia.

Y dentro de las limitaciones que tienen con respecto a las Tics son: Acceso a la red, fallas técnicas, tiempo suficiente de práctica, conocimiento de los usuarios, como las más representativas, tal como se indican en el Gráfico 2.

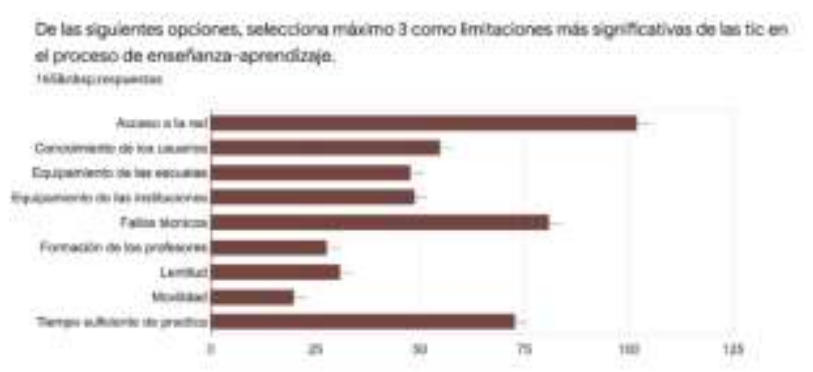

Gráfico 2 Limitaciones del uso de las Tics. Fuente: Elaboración Propia

Con respecto a los dispositivos con los que cuenta el estudiante, se identifica que solo 49 estudiantes de los encuestados no cuentan con teléfono móvil y 6 sin televisión, identificándose que estos son los dispositivos con los que los estudiantes cuentan y son las más utilizados por ellos, tal como se describe en la Grafica 3.

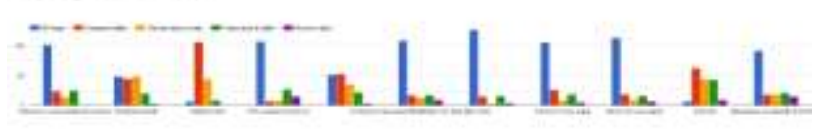

Gráfico 3 Dispositivos electrónicos con los que cuentan los estudiantes

Fuente: Elaboración Propia.

De acuerdo a las áreas donde se clasifican las competencias digitales se establece un análisis de los resultados obtenidos, considerando la relación por el sexo del estudiante. Se establecieron valores entre 2 y 10 en cada categoría como una calificación final, siendo 2 nada competente en esa área y 10 como una calificación máxima. 
En el Área 1 Información y Alfabetización, se establecieron preguntas relacionadas con el uso de los buscadores y la información que encuentran, además del almacenamiento de la información encontrada.

La Grafica 4 muestra los resultados obtenidos, donde se indica que la mediana para el sexo femenino es de 6.3 y del sexo masculino 7.5, el primer cuartil de ambos está iguales en 5.3 y el tercer cuartil es mayor en el sexo masculino. Los valores mínimos y máximo en esta categoría son 2 y 10 respectivamente.

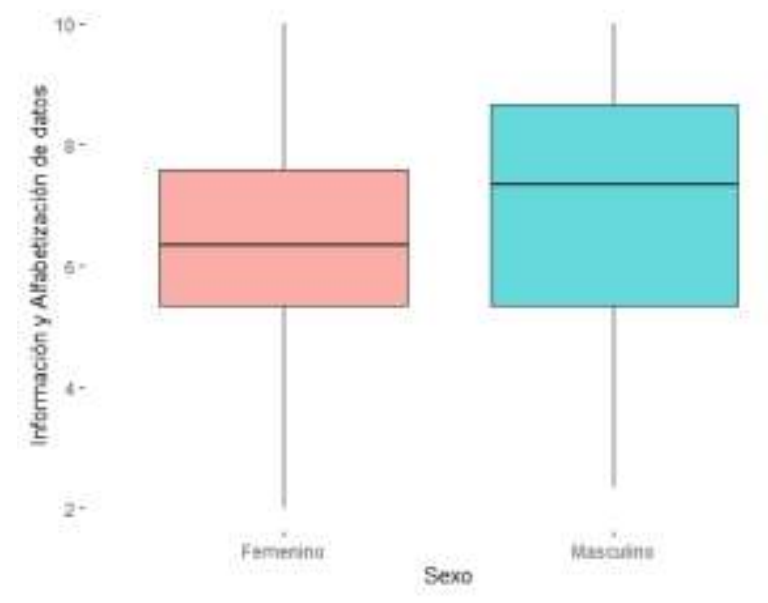

Gráfico 4 Área Información y Alfabetización por sexo Fuente: Elaboración Propia.

En el Área 2 Comunicación y Colaboración, se establecieron preguntas relacionadas con el uso de las redes sociales, medios utilizados para comunicarse y su identidad digital.

La Grafica 5 muestra los resultados obtenidos, donde se indica que las medianas son casi iguales para ambos sexos, 5.3 en el primer cuartil de los dos sexos y el tercer cuartil es un poco mayor en el sexo masculino. El valor mínimo es 4.1 y máximo 9.2, por lo que significa que los estudiantes si tienen dominio de esta competencia. Se muestra en la gráfica el caso de datos atípicos, donde se indica que hay 2 estudiantes que cuentan con la calificación de 9.2 siendo fuera del valor común del resto de 8.6, uno del sexo femenino y otro masculino.

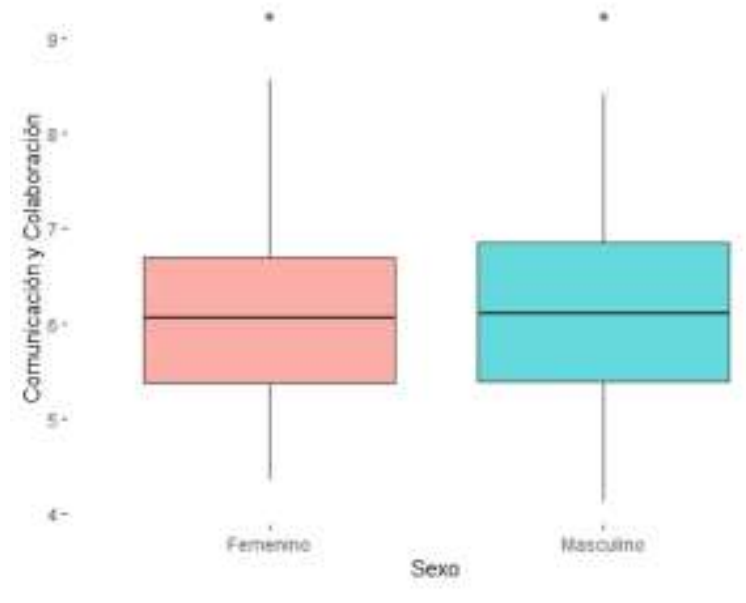

Gráfico 5 Área Comunicación y Colaboración por sexo Fuente: Elaboración Propia

En el Área 3 Creación de contenidos digitales, se establecieron preguntas relacionadas a la creación de contenidos multimedia, compartir información en diferentes comunidades virtuales, participación en entornos virtuales y el desarrollo del trabajo colaborativo.

La Grafica 6 muestra los resultados obtenidos, donde se indica que la mediana esta alrededor de 7 para ambos sexos, el primer y tercer cuartil del sexo masculino es mayor que del sexo femenino. El valor mínimo es 2.3 y máximo 10 , con un promedio de 6.9 para esta categoría.

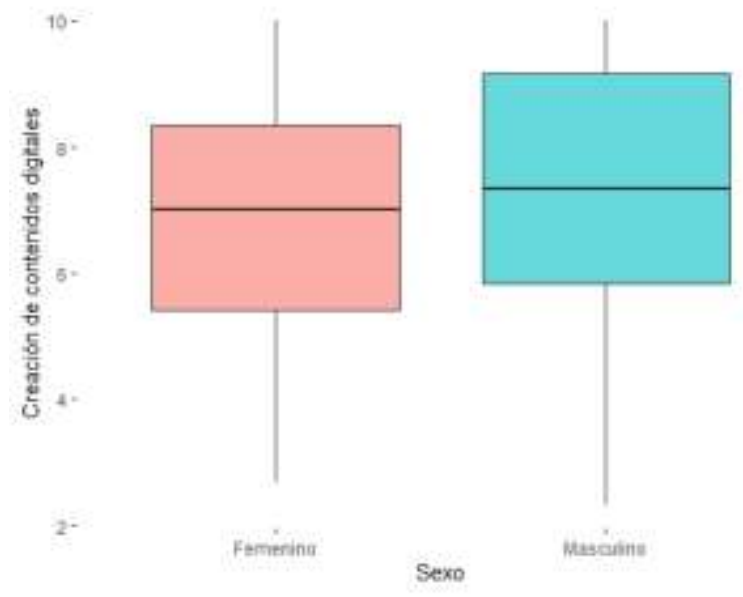

Gráfico 6 Área Información y Alfabetización por sexo Fuente: Elaboración Propia

En el Área 4 Seguridad, se establecieron preguntas relacionadas con la seguridad de sus datos personales en internet, con la protección de su información compartida, así como las medidas de seguridad física con acceso a las Tics. 
La Grafica 7 muestra los resultados obtenidos, donde se indica que la mediana es de 8.5 para el sexo femenino y de 8.6 para el masculino; el promedio es de 8.1, por lo que en esta área los alumnos tienen mayor dominio. El primer cuartil esta alrededor de 7 y un poco mayor para el sexo masculino, el tercer cuartil es de 9.5. El valor mínimo es 3, para el sexo femenino y 5.5 para el masculino, presentándose como un valor atípico, por lo que los estudiantes del sexo masculino, tienen mayor dominio en esta categoría que los del sexo femenino.

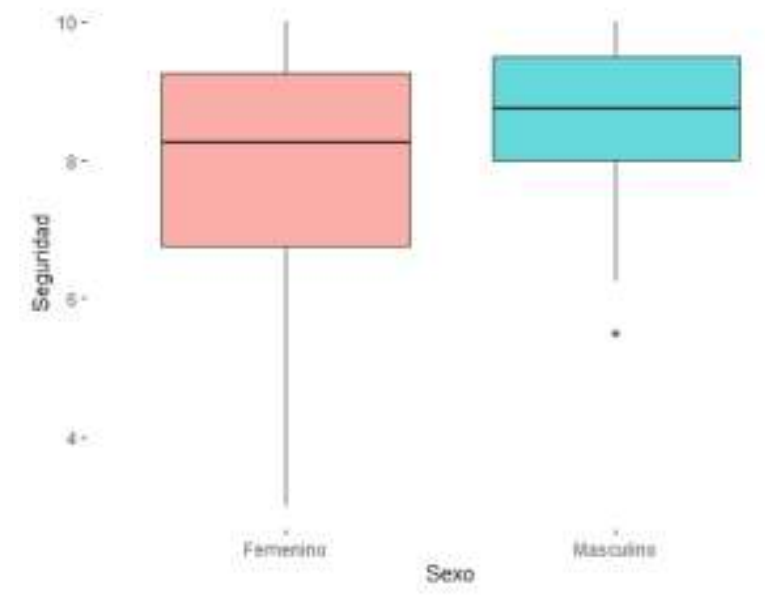

Gráfico 7 Área Seguridad.

Fuente: Elaboración Propia

En el Área 5 Resolución de problemas, se establecieron preguntas relacionadas con el conocimiento y uso de los dispositivos digitales del equipo de cómputo y la solución de problemas con el uso del mismo.

La Grafica 8 muestra los resultados obtenidos, donde se indica que la mediana es mayor en el sexo masculino con un valor de 8.6 y 7.5 para el sexo femenino, así como los valores del primer y tercer cuartil también son mayores para el sexo masculino. Los valores mínimos 2 y es mayor que del sexo femenino. Se presenta un valor atípico de 2 en el sexo masculino alejado del resto con un valor de 4.9. En el sexo femenino, también se considera un valor atípico del valor de 2 con un valor de 2.4 con el resto.

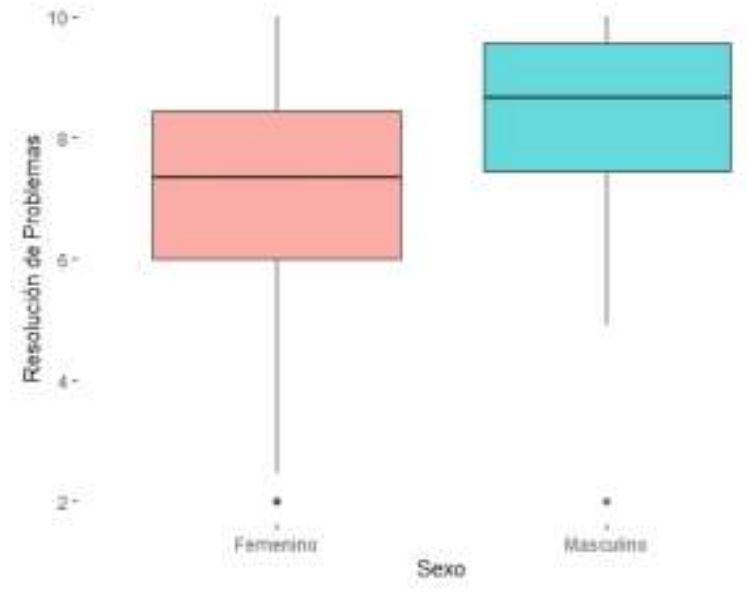

Gráfico 8 Área Resolución de Problemas por sexo. Fuente: Elaboración Propia

\section{Conclusiones}

Con los resultados obtenidos, se puede apreciar que los alumnos del sexo masculino, tienen mayor dominio de las competencias digitales que los del sexo femenino. Las competencias que más dominan los alumnos son las correspondientes al área 2 y al área 4, referentes a comunicación y seguridad.

Las áreas que se tiene con menor desarrollo son el área 1 y 5 , relacionadas con la búsqueda de información y la solución de problemas. Es de gran importancia fomentar las competencias digitales en los estudiantes, así mismo en los profesores para fortalecer el proceso enseñanza- aprendizaje.

La institución educativa no solo es de dotar de equipo y nueva tecnología a los laboratorios, sino comprometerse a un plan estratégico que como equipo fortalezca las competencias digitales de alumnos y también de los profesores.

\section{Referencias}

Almenara, J. C., \& Llorente Cejudo, M. C. (2008). La alfabetización digital de los alumnos. Competencias digitales para el siglo XXI. Revista Portuguesa De Pedagogía, (42-2), p. 728. https://doi.org/10.14195/1647-8614_42-2_1

European Commission. (2016). Communication "A New Skills Agenda for Europe - Working together to strengthen human capital, employability and competitiveness". 
European Commission. (2006).

Recommendation of the European Parliament and of the Council of 18 December 2006 on key competences for lifelong learning.

García Aretio L. (2019). Necesidad de una educación digital en un mundo digital. RIED. Revista Iberoamericana de Educación a Distancia, 22(2), pp.09-22. doi: http://dx.doi.org/10.59344/ried.22.2.233911

The Failure Institute (2018). El impacto de las competencias digitales en el emprendimiento en México. Consultado en noviembre 15, 2020 en https://thefailureinstitute.com/wp-

content/uploads/2018/11/Competencias-

digitales.pdf

UNESCO (2011). Educación de calidad en la era digital - Una oportunidad de cooperación para UNESCO en América Latina y el Caribe. OEALC/UNESCO. Argentina.

Written, I. H., \& Frank, E. (2005). Data Mining: Practical Machine learning tools and techniques. Morgan Kaufmann. 